\title{
Construction of competing endogenous RNA interaction network as prognostic markers in metastatic melanoma
}

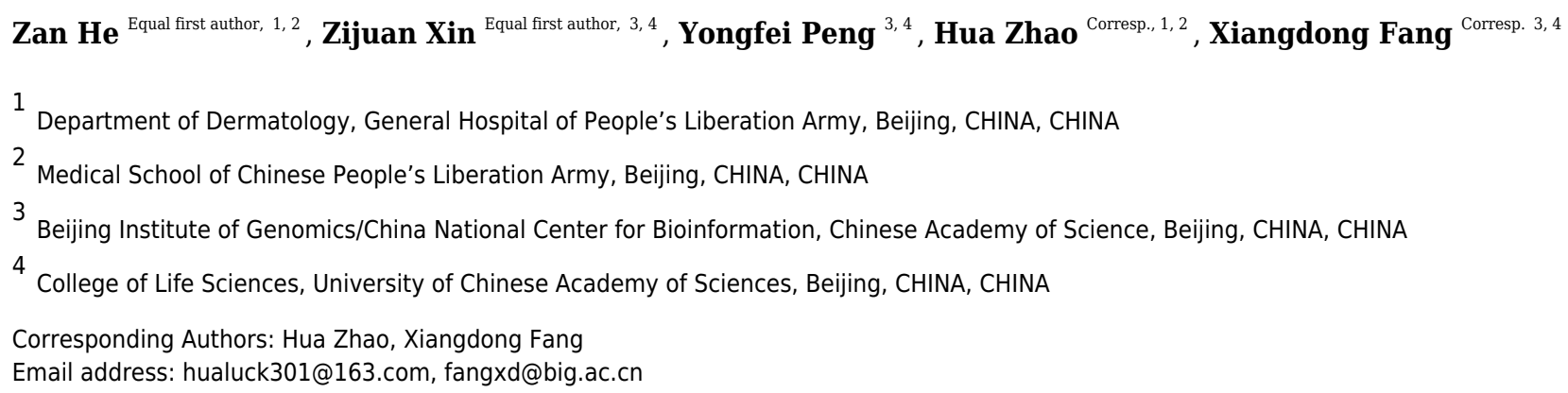

Malignant melanoma (MM) is a malignant tumor originating from melanocytes, with high aggressiveness, high metastasis and extremely poor prognosis. MM accounts for $4 \%$ of skin cancers and $80 \%$ of mortality, and the median survival of patients with metastatic melanoma is only about 6 months, with a five-year survival rate of less than $10 \%$. In recent years, the incidence of melanoma has gradually increased and has become one of the serious diseases that endanger human health. Competitive endogenous RNA (ceRNA) is the main model of the mechanism by which long chain non-coding RNAs (IncRNAs) play a regulatory role in the disease. LncRNAs can act as a "sponge", competitively attracting small RNAs (micoRNAs; miRNAs), thus interfering with miRNA function, and affect the expression of target gene messenger RNAs (mRNAs), ultimately promoting tumorigenesis and progression. Bioinformatics analysis can identify potentially prognostic and therapeutically relevant differentially expressed genes in MM, finding IncRNAs, miRNAs and mRNAs that are interconnected through the ceRNA network, providing further insight into gene regulation and prognosis of metastatic melanoma. Weighted co-expression networks were used to identify IncRNA and mRNA modules associated with the metastatic phenotype, as well as the co-expression genes contained in the modules. 17 IncRNAs, 6 miRNAs, and 11 mRNAs were used to construct a ceRNA interaction network that plays a regulatory role in metastatic melanoma patients. The prognostic risk model was used as a sorter to classify the survival prognosis of melanoma patients. Four groups of ceRNA interaction triplets were finally obtained, which miR-3662 might has potential implication for the treatment of metaststic melanoma patients, and futher experiments confirmed the regulating relationship and phenotype of this assumption. This study provides new targets to regulate metastatic process, predict metastatic potential and indicates that the miR-3662 can be used in the treatment of melanoma. 
1 Construction of competing endogenous RNA interaction networks as prognostic markers in

2 metastatic melanoma

3 Zan He Equal first author, 1, 2, Zijuan Xin Equal first author, 3, 4, Yongfei Peng 3, 4, Hua Zhao Corresp., 1, 2 ,

4 Xiangdong Fang Corresp. 3, 4

51 Department of Dermatology, General Hospital of People's Liberation Army, Beijing, CHINA, 6 CHINA

72 Medical School of Chinese People's Liberation Army, Beijing, CHINA, CHINA

83 Beijing Institute of Genomics/China National Center for Bioinformation, Chinese Academy of

9 Science, Beijing, CHINA, CHINA

104 College of Life Sciences, University of Chinese Academy of Sciences, Beijing, CHINA, CHINA

11 Corresponding Authors: Hua Zhao, Xiangdong Fang

12 Email address: hualuck301@163.com, fangxd@big.ac.cn 


\section{Abstract}

Malignant melanoma (MM) is a highly aggressive, metastatic cancer originating from melanocytes. These tumors have an extremely poor prognosis. MM accounts for $4 \%$ of skin cancers with an $80 \%$ mortality rate. The median survival of patients with metastatic melanoma is approximately six months, with a five-year survival rate of less than $10 \%$. In recent years, the incidence of melanoma has gradually increased and has become one of the deadliest cancers. Competitive endogenous RNA (ceRNA) models the mechanism by which long chain non-coding RNAs (lncRNAs) play a regulatory role in the disease. LncRNAs can act as a sponge, competitively attracting small RNAs (micoRNAs; miRNAs) and interfering with miRNA function. This can affect the expression of target gene messenger RNAs (mRNAs), ultimately promoting tumorigenesis and tumor progression. Bioinformatics analysis may identify potentially prognostic and therapeutically-relevant differentially expressed genes in MM, as well as lncRNAs, miRNAs and mRNAs that are interconnected through the ceRNA network. This may provide further insight into gene regulation and the prognosis of metastatic melanoma. Weighted coexpression networks were used to identify lncRNA and mRNA modules associated with the metastatic phenotype, as well as the co-expression genes contained in the modules. We used 17 lncRNAs, six miRNAs, and 11 mRNAs to construct a ceRNA interaction network with a regulatory role in patients with metastatic melanoma. The prognostic risk model was used as a sorter to classify the survival prognosis of melanoma patients. Four groups of ceRNA interaction triplets were obtained, and miR-3662 may be used in the treatment of metastatic melanoma patients. Experiments confirmed the regulating relationship and phenotype of this assumption. This study provides new targets to regulate the metastatic process, predict metastatic potential and determine that miR-3662 may be used in the treatment of melanoma.

\section{INTRODUCTION}

54 Malignant melanoma is derived from melanocytes, which are typically cutaneous. It is the third 
most common malignant tumor of the skin, but is the most malignant. The incidence of melanoma has continued to rise over the years, with approximately 300,000 new cases and 60,000 deaths from melanoma worldwide annually (Swick \& Maize 2012). In 2016, there were 76,380 new cases of melanoma and 10,130 patient deaths from melanoma in the United States alone (Reddy et al. 2017). Cutaneous malignant melanomas can be classified as malignant freckled nevus-like melanoma, superficial disseminated malignant melanoma, extremity malignant melanoma, and nodular malignant melanoma based on their clinical and pathologic manifestations. Metastatic melanoma accounts for $1 \%$ of all cancer cases, but many patients experience recurrence after surgery, resulting in a poor long-term prognosis and a 5-year survival rate of less than $10 \%$ (McKean \& Amaria 2018; Wahid et al. 2018). The outcomes from current adjuvant therapies based on interferon use are not satisfactory.

A better understanding of the molecular level of MM has brought attention to the use of molecular targeted therapy targeting specific genes and signaling pathways. For example, research has led to significant results in the treatment of melanoma (Eroglu et al. 2015), lymphoma (Robert et al. 2015), Merkel cell carcinoma (Engels 2019) and other tumors from relieving the immunosuppression of $\mathrm{CD} 8+\mathrm{T}$ cells in the microenvironment (Topalian et al. 2016), immunotherapy drugs for cytotoxic T lymphocyte antigen-4 (CTLA-4), and programmed cell death-1 (PD-1). However in general, the current clinical data from immunotherapy shows that its response rate is limited, and some patients have an initial drug resistance (Lei et al. 2020). The occurrence and development of the tumor is due to the combined effect of multiple gene mutations and the activation of multiple signaling pathways, thus, it is difficult to inhibit tumor proliferation simply by targeting a single site. A combined targeted therapy that focuses on multiple sites and pathways should be the focus of research in the future.

LncRNAs are non-coding RNAs with a transcript sequence length of 200 bp or more. These are closely related to the cell cycle and differentiation, development, reproduction, gender regulation, aging and numerous human diseases. MiRNAs are non-coding RNAs of 18-24 nt in length that are mainly involved in the regulation of individual development, apoptosis, proliferation and 
82

differentiation through complete or incomplete pairing with the 3 'UTR of the target genes to degrade them or inhibit their translation. LncRNAs can competitively bind miRNAs through an miRNA response element (MRE) to inhibit the negative regulation of mRNAs by miRNAs (Ala et al. 2013; Qi et al. 2015). lncRNA is of great interest among the ceRNAs and there is growing evidence that lncRNA is involved in a wide range of biological processes. LncRNAs act as molecular sponges, competing to attract miRNAs, leading to gene silencing and are involved in a variety of human diseases (Prensner \& Chinnaiyan 2011). miRNAs regulate the expression of target genes by binding to messenger RNAs (mRNAs) on target mRNAs.

The ceRNA hypothesis has attracted extensive attention in the study of the molecular biological mechanisms of cancer development. Nevertheless, a comprehensive analysis of the lncRNAmiRNA-mRNA ceRNA regulatory network based on high-throughput sequencing and large samples are still lacking in the MM field. We sought to show the specific ceRNA regulatory mechanisms in MM patients compared to non-metastatic patients, construct a ceRNA network of melanoma marker prognostic model, and provide a new perspective for elucidating the regulatory mechanisms and prognosis of metastatic melanoma by extracting the lncRNA, miRNA, and mRNA expression matrices of skin cutaneous melanoma (SKCM) in situ and metastatic samples from the TCGA database, and obtaining the hub genes.

\section{MATERIALS \& METHODS}

All bioinformatics analyses were conducted using RStudio (R-4.0.0). Our analyses and study design are shown in Figure 1A. The interaction networks of Figure S2 and Figure S3 were drawn using Cytoscape 3.7.

\section{Patient cohort information and data processing}

The melanoma patient cohort data were obtained from the Cancer Genome Atlas (TCGA), which is deposited on the GDC website (https://portal.gdc.cancer.gov/repository). Three files were downloaded containing miRNA, isoform, and clinical information, respectively. A total of 470 melanoma samples were stored in the database, including 103 in situ melanoma samples and 367 
109 metastatic melanoma samples. The data were downloaded using the "download" command from

110 the recommended download tool gdc-client (https://gdc.cancer.gov/access-data/gdc-data-

111 metastatic-tool). The clinical information and miRNA expression matrix were obtained using the

112 R package "XML". mRNA and lncRNA were extracted separately according to the genome

113 annotation file. The mRNA extraction parameters were type = "gene", gene_biotype =

114 "protein_coding", and gene_biotype = "protein_coding", the lncRNA extraction parameters

115 arguments were type = "transcript", transcript_biotype = "lncRNA".

116 "3prime_overlapping_ncRNA", "bidirectional_promoter_lncRNA", "sense_". overlapping",

117 "sense_intronic" and "antisense_RNA".

118 Identification of differentially expressed genes

119 We used the R package "edgR" to identify differential genes between metastatic and in situ

120 melanoma samples (Robinson et al. 2010). All q-values were corrected for statistical significance

121 of multiple testing using FDR, with screening thresholds of $|\log 2 \mathrm{FC}|>1.0, \mathrm{FRD}<0.05$, and retention

122 of significantly different genes (Lai 2017; McCarthy et al. 2012).

\section{Functional enrichment analysis}

124 ClusterProfiler was used for GO/KEGG analysis (Gene Ontology/ Kyoto Encyclopedia of Genes 125 and Genomes) (Ashburner et al. 2000; Kanehisa et al. 2010; Yu et al. 2012). GO was used to 126 describe gene functions along three aspects: biological process (BP), cellular component (CC), 127 and molecular function (MF). KEGG was searched for pathways at the significance level of $128 \mathrm{p}<0.05$.

\section{Weighted correlation network analysis}

130 WGCNA is an algorithm used for the identification of gene co-expression networks for mRNAs 131 or lncRNAs with different characteristics by high-throughput spectrum of expression. 132 Neighborhood matrix assessment of weighted co-expression relationships between all datasets was 133 performed using Pearson's correlation analysis. We used WGCNA to analyze mRNAs and 134 lncRNAs to obtain the mRNAs and lncRNAs with which the metastatic melanoma samples were 135 most associated. 
136

137

138

139

140

141

142

143

144

145

146

147

148

149

150

151

152

153

154

155

156

157

158

159

160

161

162

\section{Construction of a ceRNA regulatory network}

Some authoritative databases have provided relationship pairs such as lncRNA-miRNA and miRNA-mRNA, which can assist in the construction of a ceRNA network centered on miRNA, based on an experimental module and prediction module. MirDIP (http://ophid.utoronto.ca/mirDIP/index.jsp) was used to predict interactions between lncRNAs and miRNAs.

LncBase

(http://carolina.imis.athena-

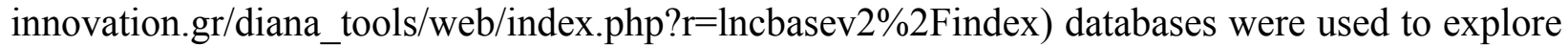
target mRNAs.

\section{Construction of metastatic melanoma survival risk prognosis model}

Metastatic melanoma samples with low expression of miRNA, high expression of lncRNA, and mRNA in the ceRNA network were used to perform three LASSO regression modeling filters. Factors that fit the normalized lambda value model were included in the subsequent analysis. A prognostic model was constructed using multiple COX regression analyses, and a comprehensive prognostic scoring system was obtained based on these factors. The risk score was calculated as follows: risk score $=\sum \beta i \times \operatorname{expRNAi}$, where expRNA is the expression level of RNA and $\beta$ is the regression coefficient derived from the multivariate Cox regression model. Melanoma patients were classified into high-risk or low-risk groups based on the median risk score cutoff value based on this risk score formula and other factors we determined. Kaplan-Meier analysis was used to determine the overall survival between the groups and there was a difference in overall survival (OS) between the two groups. $\mathrm{P}<0.05$ was considered significant unless otherwise stated.

\section{Cell line and cell culture}

A375 (ATCC, Manassan, VA, USA, Catalog number: CRL-1619) cells were cultured in an incubator in Dulbecco's modified Eagle's medium (DMEM, Gibco, Grand Island, NY, USA) containing 10\% fetal bovine serum (AusGeneX, Molendinar, Qld, Australia) and 1\% penicillinstreptomycin (P/S; Invitrogen, Carlsbad, CA, USA) with a humidity of $5 \% \mathrm{CO}_{2}$ at $37^{\circ} \mathrm{C}$.

Fluorescence in situ hybridization(FISH)

The A375 cells were inoculated in a 12-well plate and incubated overnight in an incubator set at 
$37^{\circ} \mathrm{C}$ with $5 \% \mathrm{CO} 2$. The medium in the orifice plate was absorbed and washed twice with PBS, and then $1 \mathrm{ml} \mathrm{4 \%}$ paraformaldehyde was added to each well, and fixed at room temperature for 15 min. We followed the manufacturer's instructions for the RNA FISH kit (GenePharma, Suzhou, China, Catalog number: F12201/50). Five probes were designed and the sequences are listed in Supplementary Table 1 . We added $200 \mu$ diluted DAPI working solution to each well, and stained the samples for 20 min in dark. An anti-quenching agent was added to a clean slide, and these were observed under a fluorescence microscope (630×, oil lens).

\section{Plasmid, siRNA and primer synthesize}

Plasmids of miRNA-has-miR-3662 and miRNA-shNC, siRNA of siRP11-594N15.3 and si-NC were bought from GenePharma. The overexpressed plasmids of the miR-3666 sequence are shown in Supplementary Table 2. The primers of RP11-594N15.3, ZNF831, PKIA, CSF2RB, miR-3662, GAPDH, U6 and reverse universal primer were designed by Primer Premier 5 software and were purchased from the Beijing Genomics Institute (Supplementary Table 3).

\section{Expression vectors transfection}

A375 cells were cultured in $10 \mathrm{~cm}$ petri dishes at a concentration of $7 \times 10^{6} / 8 \mathrm{ml}$ the day after transfection. Cells were stably transduced with the miR-3662 and shNC expression vectors, using Lipofectamine 2000 (Invitrogen) according to the manufacturer's protocol. Each plate was added to $12 \mu \mathrm{g}$ plasmids and $24 \mu \mathrm{l}$ Lipofectamine 2000 . The medium was replaced after $6 \mathrm{~h}$ of transfection. Each plate was added to $80 \mu \mathrm{M}$ siRNA for the transfection of siRNA and additional steps were performed as described above.

\section{RNA isolation and quantitative real-time PCR (qRT-PCR)}

We used TRIzol® Reagent (Life Technologies) to extract RNA from cells. After wiped off DNA, equal amounts $(2 \mu \mathrm{g})$ of each RNA were separately reverse transcribed to their corresponding cDNAs using a RevertAid ${ }^{\mathrm{TM}}$ First Strand cDNA Synthesis Kit (Thermo Fisher Scientific). All the opeations are subject to the manufacturer's instructions. CFX96 Real-Time PCR detection system (Bio-Rad) was used to perform qRT-PCR with Maxima ${ }^{\circledR}$ SYBR Green/ROX qPCR Master Mix (Thermo Fisher Scientific). U6 and GAPDH were used as internal control for miR-3662 and other 
mRNAs respectively.

\section{Cell counting kit-8 (CCK-8) assay}

2,000 cells/well were seeded into 96-well plates, and were cultured for one, two, three, or four days. The condition of cell proliferation was assessed with a Cell Counting Kit-8 assay (C0038, Beyotime Biotechnology, China). According to manufacturer's instruction, cells were treated with $10 \mu \mathrm{L}$ CCK-8 (100 $\mu \mathrm{L}$ culture medium per well) solution for $2 \mathrm{~h}$ at $37^{\circ} \mathrm{C}$ after a period of incubation. Absorbance at $450 \mathrm{~nm}$ was measured by using a microplate reader.

\section{Transwell migration and invasion assays}

The cells were resuspended in serum-free medium the day before experiments. For Transwell invasion assays, Transwell upper chambers $(8 \mathrm{~mm}$, BD Biosciences, Franklin Lakes, BJ, USA) were coated with Matrigel matrix (dilution 1:7; 356234, BD Biosciences) and $2 \times 10^{5}$ cells were placed in. For Transwell migration assays, $2 \times 10^{4}$ cells were seeded in the upper chamber of the Transwell without Matrigel matrix. Then, a medium containing 20\% serum was added to lower chambers. Cells were incubated at $37^{\circ} \mathrm{C}$ and $5 \% \mathrm{CO} 2$ for $18 \mathrm{~h}$, and then those cells remaining in upper chambers were removed using a wet cotton swab. We used $4 \%$ paraformaldehyde to fix the cells that had migrated and adhered to lower chambers for $10 \mathrm{~min}$. Then hematoxylin (ZLI-9610, ZSGB-Bio) and eosin (ZLI-9613, ZSGB-Bio) were used to stain cells adhered to lower chambers for $20 \mathrm{~min}$ and then imaged. The number of cells was counted in ten separated high power fields with vertical cross distribution.

\section{RESULTS}

\section{Identification of differential genes in metastatic and in situ melanoma samples}

There were 103 samples of melanoma in situ and 367 samples of metastatic melanoma in the TCGA database (Supplementary Table 4, Supplementary Table 5). We identified 1,413 differential lncRNAs, of which 1,059 were highly-expressed in metastatic samples and 354 in the in-situ samples. We identified 2,251 differential mRNAs, of which 1,442 were highly-expressed in the metastatic samples and 809 in the in-situ samples. We identified 81 differential miRNAs, of which 
21760 were highly expressed in metastatic samples and 21 in the in-situ samples (Figure 1B-1D, 218 Figure S1 A-C). The differentially expressed mRNAs in the metastatic group were enriched for

219 GO function and KEGG pathway, immune cell activity-related terms on biological processes (BP), 220 transmembrane transduction structures on cellular composition (CC), G protein-coupled receptor

221

222

223

224

225

226

227

228

229

230

231

232

233

234

235

236

237

238

239

240

241

242

243

activity, passive transport and other entries on molecular function (MF) (Figure 1E). The KEGG pathway was enriched for the PI3K-Akt signaling pathway, chemokine signaling pathway, JAKSTAT signaling pathway, cell adhesion molecules (CAMs), allograft rejection, NF-kappa-B signaling pathway, and other tumor-related pathways (Figure 1F).

\section{Identification of mRNA and IncRNA co-expression modules by weighted gene co-expression} network analysis

We used weighted gene co-expression network analysis (WGCNA) to analyze the gene expression patterns of mRNA and lncRNA expression matrices extracted from metastatic and in-situ samples.

Genes with similar expression patterns were clustered into a co-expression module (Langfelder \& Horvath 2008). We identified nine mRNA co-expression modules and 10 lncRNA co-expression modules in the two groups, and the number of genes contained in each module is shown in Supplementary Table 6 . The significantly related co-expression modules were selected according to the clustering of linkage levels and the number of co-expression associations. The mRNA clustering results showed that the black module and yellow module of in-situ samples, and the turquoise module of metastatic samples were identified as highly-associated co-expression modules (Figure 2A-C). The lncRNA clustering results showed that the pink and red module of in-situ samples, and the turquoise and brown modules of the metastatic samples were identified as highly correlated co-expression modules (Figure 2D-F). The corresponding interactions of the first 20 co-expressed genes in the above modules are shown in Figure S2 A-G. GO functional enrichment of co-expression genes in the turquoise module in mRNA showed that the module genes were associated with immune responses, and were also enriched for entries associated with immune negative regulation and cell adhesion (Figure 2G). We removed the turquoise module in mRNA and the brown/turquoise module in lncRNA for the next step of the analysis. 
244

245

246

247

248

249

250

251

252

253

254

255

256

257

258

259

260

261

262

263

264

265

266

267

268

269

270

\section{Construction of ceRNA regulation network}

The mRNAs from the turquoise module obtained after WGCNA analysis were intersected with the highly expressed mRNAs in the metastatic samples, and 319 mRNAs were obtained. lncRNAs from the brown/turquoise module were intersected with the highly expressed mRNAs in the metastatic samples, and 287 lncRNAs were obtained (Fig. 3.A-B). The above 319 mRNAs were subjected to Pearson's correlation coefficient calculation with 287 lncRNAs. The results showed that 138 mRNAs had potential interactions with 89 lncRNAs when cor $>0.5$ and pvalue $<0.05$ (Figure S3). Using the mirDIP database to predict the target genes, the low expression miRNAs in the 21 metastatic samples were predicted to score "very high" of 3,771 mRNA target genes. The 3,771 target genes were cross-linked to 138 mRNAs by correlation analysis to obtain 11 mRNAs (CD53, CD96, CSF2RB, CXCL9, MS4A1, PIM2, PKIA, PTPN22, SH2D1A, SLA, ZNF831) (Figure 3C). The 11 mRNAs and their corresponding six miRNAs (has-miR-346, has-miR-3662, has-miR-429, has-miR-891b, has-miR-892a, has-miR-944) were analyzed and mapped for the interaction network (Figure 3D). These six miRNAs were used to predict 3,760 related lncRNAs in the lncBase database. The 3,760 lncRNAs were intersected with $89 \ln R$ NAs screened in correlation analysis to obtain 17 lncRNAs (AC007386.4, AC104024.1, GTSCR1, LINC00402, LINC00861, MIAT, PRKCQ-AS1, RP11-121A8.1, RP11-164H13.1, RP11-202G28.1, RP11203B7.2, RP11-594N15.3, RP11-598F7.3, RP11-693J15.5, RP11-768B22.2, RP5-887A10.1) (Figure 3E). Interaction networks were mapped for the above 17 lncRNAs and six miRNAs (Figure 3F). The final selection of $11 \mathrm{mRNAs}$ and lncRNAs, the data tSNE downscaling analysis, were better able to distinguish the in-situ samples from the metastatic samples (Figure 3G), resulting in the construction of InRNA-miRNA-mRNA ceRNA network with the final selection of six miRNAs (Figure 3H). The factors of our network were found to be highly expressed in metastatic samples in another melanoma cohort (GEO database; accession number: GSE65904) (Figure 3I).

\section{Construction of metastatic melanoma survival risk prognosis model}

We clustered the 34-factors included in our ceRNA interaction network, and 17 lncRNAs, 6 miRNAs, and 11 mRNAs were subjected to risk prediction by LASSO regression, respectively. 
271 The regularization term controlled for the lncRNA, miRNA, and mRNA models having minimal 272 likelihood error when $\lambda=7,1$, and 2, respectively. We screened lncRNAs, one miRNA, and two 273 mRNAs for high correlation with prognosis (Figure 4A-F). The above 10 factors were analyzed 274 using multiple COX regression to construct risk models. There were five factors considered to be risk factors for prognosis and the other five factors were protective factors for prognosis. lncRNA AC104024.1, MIAT, RP11-598F7.3, and miRNA hsa-mir-429 could be used as significant risk markers $(\mathrm{P}<0.05)$. The concordance index of the regression model was 0.753 (Figure $4 \mathrm{G})$. In the cohort, the expressions of AC104024.1, AC007386.4, and has-mir-429 were gradually upregulated as the risk scoring increased, while the expressions of RP11-598F7.3, PKIA, CXCL9 and MIAT were down-regulated (Figure 4H). The KM curves of melanoma patient survival were plotted according to the expression profiles of risk and protective factors in the multivariate COX regression model. These showed that the altered expression of factors in the model was significantly correlated with the follow-up prognosis of the cohort of patients $(\mathrm{P}<0.0001)$ (Figure 4I). Four groups of ceRNA interacting triplets were obtained (RP11-594N15.3-miR3662CSF2RB，RP11-594N15.3-miR3662-ZNF831，RP11-594N15.3-miR3662-PKIA，AC104024.1miR346-PKIA), of which miR3662 may have potential for the treatment of metastatic melanoma (Figure 4J).

miR-3662 down-regulates its target mRNAs and suppresses melanoma cell proliferation, migration and invasion in vitro

In order to verify whether the regulatory relationship of ceRNA was objective, we chose three groups of ceRNA interacting triplets (RP11-594N15.3-miR3662-CSF2RB, RP11-594N15.3miR3662-ZNF831，RP11-594N15.3-miR3662-ZNF831，RP11-594N15.3-miR3662-PKIA) for experimental verification. The localization of lncRNA in the cytoplasm is the premise of its spongy function. The database "IncBase" was used to predict the interaction of lncRNA-miRNA based on a base experimental module and prediction module. The binding sites on the sequence between lncRNAs and miRNAs, and the interaction between lncRNAs and miRNAs was tested in other studies. Since miRNAs only exist in the cytoplasm, the lncRNAs predicted by the database must 
298

299

300

301

302

303

304

305

306

307

308

309

310

311

312

313

314

315

316

317

318

319

\section{0}

321

322

323

324

also theoretically exist in the cytoplasm. We conducted FISH experiment in melanoma A375 cells and selected lncRNA RP11-594N15.3 for localization to ensure that our lncRNAs conformed. Our results show that RP11-594N15.3 may be localized in the cytoplasm (Figure 5A). Since RP11$594 \mathrm{~N} 15.3$ is an RNA with a length of more than $3 \mathrm{~KB}$, it is very difficult to construct its overexpression vector. Therefore, we decided to knock down the expression of RP11-594N15.3 with siRNA in A375 cells to observe the changes in the expression of miR-3662, CSF2RB, PKIA and ZNF831 (Figure 5B). miR-3662 was found to be significantly overexpressed, PKIA and ZNF831 were down-regulated, and CSF2RB was not significant (Figure 5C, D). Our experiments proved that the sponge function of lncRNA was objective.

In order to confirm whether the regulation of miR-3662 on target mRNAs in ceRNA regulatory triplets was objective, we transfected the miR-3662 overexpression plasmid vector and the NC plasmid vector into melanoma A375 cells, obtained A375-miR3662 and A375-NC cells, and verified the high expression of miR-3662 at the transcriptional level (Figure 5E). The qRT-PCR of the downstream target mRNAs shows the transcriptional differences of the three targets. The transcriptional levels of PKIA and ZNF831 were down-regulated by miR-3662, but there was no significant change on CSF2RB (Figure 5F). The overexpression of miR-3662 significantly promoted cell proliferation (Figure 5G). We used the Transwell chamber assay to explore the functions of miR-3662 on the invasion and migration of melanoma cells. The overexpression of miR-3662 significantly suppressed cell migration through a permeable filter and invasion through Matrigel Matrix (Figure 5H). Our results indicate that miR-3662 may repress melanoma cell proliferation, migration and invasion by down-regulating PKIA and ZNF831.

\section{DISSCUSSION}

The clinical treatment of melanoma poses a significant health and economic burden. There is a need to develop new chemotherapy drugs, surgical procedures and patient care (Meng et al. 2018). The ability of tumor cells to migrate and metastasize is an important feature in the pathogenesis of melanoma. The accurate diagnosis of early primary melanoma and timely surgical resection can 
325

326

327

328

329

330

331

332

333

334

335

336

337

338

339

340

341

342

343

344

345

346

347

348

349

350

351

significantly improve patient survival. However, the long-term survival of patients with metastatic melanoma is very low (Filippi et al. 2016). In this complex regulatory process, mRNAs in the tumor cell and the proteins they encode are aberrant, and the non-coding RNAs and miRNAs involved as upstream regulators also show specificity in their expression profiles, while lncRNAs, as non-coding RNAs, are involved in regulating gene and protein expression at the epigenetic level (Wilusz et al. 2009). It has been phenotypically demonstrated that miRNAs can influence the progression of melanoma by inhibiting mRNA. For example, miR-331 can inhibit the proliferation and metastasis of melanoma cells by downregulating AEG-1 (Chen et al. 2018a).

We used The Cancer Genome Atlas (TCGA) database to obtain bioinformatics that were used to construct a differentiated endogenous competitive RNA network among patients with melanoma metastasis. We conducted a risk assessment of key factors in the network to find prognostic markers. The Weighted Gene Co-expression Network Analysis (WGCNA) was used to find gene modules for collaborative expression and to explore the relationships between gene networks and phenotypes of interest. We selected the highly expressed ceRNA in the metastatic samples after the intersection of the genes and differential genes in the co-expression module recognized by WGCNA. The lncRNA and miRNA were predicted through the mirDIP and lncBase database, and the interactive relationship between miRNA and mRNA was established to construct the overall ceRNA interaction network of DElncRNA-DEmiRNA-DEmRNA in the metastatic melanoma samples. The 17 highly expressed DElncRNAs were selected from metastatic melanoma patients and triggered the down-regulation of the expression of their corresponding 6 DEmiRNAs by exerting their sponge function. This led to the high expression of the 11 target gene DEmRNAs in metastatic patients and exerted a regulatory effect on the proliferation and invasion of melanoma cells in the process of transcription. We provided a new perspective by elucidating the upstream molecular mechanisms of melanoma invasion and migration.

We conducted three LASSO regression modeling screening of 34 factors according to lncRNA, miRNA and mRNA, respectively, in order to explore the correlation between factors in the ceRNA network and the prognosis of the cohort samples. The 10 factors were screened for best fit to the 
352 model with regularized lambda values. The risk factors of the 10 factors were modeled using 353 multiple COX regression, and the results showed that three lncRNAs (AC104024.1, RP11354 594N15.3, MIAT), one miRNA (miR-429), one mRNA (PKIA) were identified as molecular 355 markers with high correlation with patient prognosis by constructing the risk model. AC104024.1, 356 RP11-594N15.3, and miR-429 were risk factors for prognosis; MIAT PKIA was a protective factor 357 for prognosis. The above factors may be used as markers to predict metastatic potential in 358 melanoma patients and may also serve as a target to distinguish the survival prognosis of 359 melanoma patients. Finally, we screened four groups of ceRNA regulatory triplets present in 360 metastatic melanoma patients, in which AC104024.1 and RP11-594N15.3 served as key 361 endogenous sponges that upregulated the expression level of target genes CSF2RB, ZNF831, and 362 PKIA by competitive adsorbing miR-364 and miR-3662, respectively. Our assays in the A375 cell 363 line confirmed that the phenotype of miR-3662 was consistent with the anticipation of 364 bioinformatics analysis. The candidate genes above were studied related to cancer progression. 365 Zhu et al. (2019) expressed miR-3662 through a lentiviral vector, which could significantly reduce 366 the ZEB1 protein level and inhibit the growth of A375 cells in vitro and in vivo. The expression of ZEB1 induced by miR-3662 decreased, the EMT expression of A375 cells was inhibited, and the relative expression of metastatic genes was reduced. Down-regulation of ZEB1 expression by a miR-3662 lentivirus vector may significantly reduce in vitro and in vivo growth of the highly invasive melanoma cell line A375. miR-3662 was considered to be a therapeutic target in adult Tcell leukemia/lymphoma (ATLL) studies. miR-3662 knockdown inhibited the proliferation of ATLL cells, and the expression of miR-3662 was related to the antiviral resistance of ATLL cells (Yasui et al. 2018). miR-3662 in hepatocellular carcinoma tends to be downregulated, and the high expression of miR-3662 inhibits hepatocellular carcinoma growth by inhibiting HIF-1 $\alpha$ (Chen et al. 2018b). miR-429 also plays a regulatory role in the development of various tumors. For example, it promotes the proliferation of bladder cancer cells by inhibiting CDKN2B (Yang et al. 2017). ZEB1 expression was regulated to promote the migration, invasion and EMT of pancreatic cancer (Shen et al. 2019). miR-429 may also promote the proliferation of non-small cell lung 
379

380

381

382

383

384

385

386

387

388

389

390

391

392

393

394

395

396

397

398

399

400

401

402

403

404

405

cancer by targeting DLC-1 (Xiao et al. 2016). Our study showed that in patients with metastatic melanoma, the low expression of microRNAs, miR-3662 and miR-429, led to the upregulation of the expression of target genes, CSF2RB, ZNF831and PKIA, while the transcription of differentially expressed mRNA played a specific role in the tumor phenotype.

CSF2RB (Colony Stimulating Factor 2 Receptor Subunit Beta) is involved in multiple signaling pathways, and promotes survival, proliferation, and differentiation through JAK2/STAT5, $\mathrm{PI3K} / \mathrm{mTOR}, \mathrm{MEK} / \mathrm{ERK}$, and other signaling pathways (Hercus et al. 2013). Cimas et al. (2020) identified CSF2RB mutations in patients with high PD-1/PD-L1 expression using the RNA-seq of basal-like breast tumors, combined with the TCGA database, suggesting good prognosis and correlation with immune infiltration. In a public database study of lung adenocarcinoma, Xu et al. (2020) identified nine genes, including CSF2RB, from three independent LUAD cohorts (Xu et al. 2020). In colorectal cancer patients, CSF2RB was matched with 10 miRNAs that were involved

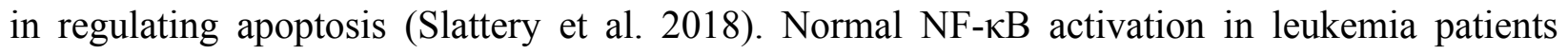
positively regulated the expression of IL-3 and granulocyte/macrophage CSF2RB, thereby promoting the proliferation and survival of CML stem cells. No studies have been conducted, to our knowledge, on CSF2RB in melanoma. The protein encoded by PKIA (CAMP-Dependent Protein Kinase Inhibitor Alpha) is a member of the cAMP-dependent protein kinase (PKA) inhibitor family. Zhang et al. (2020) analyzed the gene transcriptome profiles of eight GEO cohorts in thyroid cancer, performed LASSO regression models, and identified seven genes, including PKIA, that were highly correlated with recurrence data in the TCGA database (Zhang et al. 2020). In the field of cervical cancer research, PKIA is considered an important marker for stage III cervical cancer (Banerjee \& Karunagaran 2019). Hoy et al. (2020) found that the amplification of PKIA is common in prostate cancer and was associated with reduced progression-free survival. Depletion of PKIA in prostate cancer cells results in reduced migration, increased sensitivity to nest loss apoptosis, and reduced tumor growth. By altering the activity of protein kinase A, protein kinase I can act as a molecular switch that drives the gPCR-GMAP1 s-cAMP signal toward activation of $\alpha$-RAP1 and MAPK, ultimately regulating tumor growth (Hoy et al. 2020). As a 
406

407

408

409

410

411

412

413

414

415

416

417

418

419

420

421

422

423

424

425

426

427

428

429

430

431

432

transcription factor, ZNF831 (Zinc Finger Protein 831) is thought to regulate the immune response network with phenotypes in breast cancer (da Silveira et al. 2017). The co-expression network was also identified in the H-immune subtype of triple negative breast cancer (He et al. 2018). ZNF831 may regulate tumor immune infiltration in melanoma and plays an important role in infiltration and immune escape from melanoma.

There are some limitations to our study although we provided a complete analysis, used reasonable analytical tools, verified our results by phenotypic assays, and the results are clinically important for elucidating the regulatory mechanism and prognosis of melanoma metastasis. We predicted the interaction network of ceRNA through the database, selected RP11-594N15.3-miR-3662 and its target mRNAs for verification, however, we failed to verify all the regulatory relationships in the network objectively in vitro. We constructed a risk classification model, but did not verify the universality of the model in the verification set because it was difficult to find another melanoma queue containing lncRNA and miRNA sequencing data. Finally, 2-lncRNAs in the four groups of triplets lacked the evidence of previous relevant studies in tumors. Future studies should explore its function and mechanism.

\section{CONCLUSIONS}

We used the differentially expressed genes in situ and metastatic samples of melanoma, as well as the phenotypic co-expression module of metastatic melanoma to construct a ceRNA interaction network that may regulate melanoma metastasis. We established a classifier model that can predict the prognosis of melanoma patients through LASSO regression and multivariate COX regression. We found that AC104024.1 and RP11-594N15.3 can be used as markers in patients with melanoma, indicating a poor prognosis. We obtained four groups of ceRNA regulatory triplets present in metastatic melanoma patients, in which AC104024.1 and RP11-594N15.3 served as key endogenous sponges that upregulated the expression level of target genes CSF2RB, ZNF831, and PKIA by competitive adsorbing miR-346 and miR-3662, respectively. These may regulate the metastasis of melanoma and lead to a worse prognosis. Further experiments have shown that miR- 
433

434

435

436

437

438

439

440

441

442

443

444

445

446

447

448

449

450

451

452

453

454

455

456

457

458

459

460

461

462

463

464

465

466

467

468

469

470

3663 can reduce the transcription of its target mRNAs, and that it inhibits the proliferation, invasion and migration of melanoma cells.

\section{References}

Ala U, Karreth F, Bosia C, Pagnani A, Taulli R, Léopold V, Tay Y, Provero P, Zecchina R, and Pandolfi P. 2013. Integrated transcriptional and competitive endogenous RNA networks are cross-regulated in permissive molecular environments. Proceedings of the National Academy of Sciences of the United States of America 110:7154-7159. 10.1073/pnas.1222509110

Ashburner M, Ball C, Blake J, Botstein D, Butler H, Cherry J, Davis A, Dolinski K, Dwight S, Eppig J, Harris M, Hill D, Issel-Tarver L, Kasarskis A, Lewis S, Matese J, Richardson J, Ringwald M, Rubin G, and Sherlock G. 2000. Gene ontology: tool for the unification of biology. The Gene Ontology Consortium. Nature genetics 25:25-29. 10.1038/75556

Banerjee S, and Karunagaran D. 2019. An integrated approach for mining precise RNA-based cervical cancer staging biomarkers. Gene 712:143961. 10.1016/j.gene.2019.143961

Chen L, Ma G, Cao X, An X, and Liu X. 2018a. MicroRNA-331 inhibits proliferation and invasion of melanoma cells by targeting astrocyte-elevated gene-1. Oncology research. 10.3727/096504018x15186047251584

Chen Z, Zuo X, Zhang Y, Han G, Zhang L, Wu J, and Wang X. 2018b. MiR-3662 suppresses hepatocellular carcinoma growth through inhibition of HIF-1 $\alpha$-mediated Warburg effect. Cell death \& disease 9:549. 10.1038/s41419018-0616-8

da Silveira W, Palma P, Sicchieri R, Villacis R, Mandarano L, Oliveira T, Antonio H, Andrade J, Muglia V, Rogatto S, Theillet C, du Manoir S, and Tiezzi D. 2017. Transcription Factor Networks derived from Breast Cancer Stem Cells control the immune response in the Basal subtype. Scientific reports 7:2851. 10.1038/s41598017-02761-6

Engels EA. 2019. Epidemiologic perspectives on immunosuppressed populations and the immunosurveillance and immunocontainment of cancer. Am J Transplant 19:3223-3232. 10.1111/ajt.15495

Eroglu Z, Kim DW, Wang X, Camacho LH, Chmielowski B, Seja E, Villanueva A, Ruchalski K, Glaspy JA, Kim KB, Hwu WJ, and Ribas A. 2015. Long term survival with cytotoxic T lymphocyte-associated antigen 4 blockade using tremelimumab. Eur J Cancer 51:2689-2697. 10.1016/j.ejca.2015.08.012

Filippi A, Fava P, Badellino S, Astrua C, Ricardi U, and Quaglino P. 2016. Radiotherapy and immune checkpoints inhibitors for advanced melanoma. Radiotherapy and oncology : journal of the European Society for Therapeutic Radiology and Oncology 120:1-12. 10.1016/j.radonc.2016.06.003

He Y, Jiang Z, Chen C, and Wang X. 2018. Classification of triple-negative breast cancers based on Immunogenomic profiling. Journal of experimental \& clinical cancer research : CR 37:327. 10.1186/s13046-018-1002-1

Hercus T, Dhagat U, Kan W, Broughton S, Nero T, Perugini M, Sandow J, D'Andrea R, Ekert P, Hughes T, Parker M, and Lopez A. 2013. Signalling by the $\beta \mathrm{c}$ family of cytokines. Cytokine \& growth factor reviews 24:189201. 10.1016/j.cytogfr.2013.03.002

Peer] reviewing PDF | (2020:12:56394:1:1:NEW 27 Jul 2021) 
Hoy J, Salinas Parra N, Park J, Kuhn S, and Iglesias-Bartolome R. 2020. Protein kinase A inhibitor proteins (PKIs) divert GPCR-Gas-cAMP signaling toward EPAC and ERK activation and are involved in tumor growth. FASEB journal : official publication of the Federation of American Societies for Experimental Biology. 10.1096/fj.202001515R

Kanehisa M, Goto S, Furumichi M, Tanabe M, and Hirakawa M. 2010. KEGG for representation and analysis of molecular networks involving diseases and drugs. Nucleic acids research 38:D355-360. 10.1093/nar/gkp896

Lai Y. 2017. A statistical method for the conservative adjustment of false discovery rate (q-value). $B M C$ bioinformatics 18:69. 10.1186/s12859-017-1474-6

Langfelder P, and Horvath S. 2008. WGCNA: an R package for weighted correlation network analysis. $B M C$ bioinformatics 9:559. 10.1186/1471-2105-9-559

Lei Q, Wang D, Sun K, Wang L, and Zhang Y. 2020. Resistance Mechanisms of Anti-PD1/PDL1 Therapy in Solid Tumors. Front Cell Dev Biol 8:672. 10.3389/fcell.2020.00672

McCarthy D, Chen Y, and Smyth G. 2012. Differential expression analysis of multifactor RNA-Seq experiments with respect to biological variation. Nucleic acids research 40:4288-4297. 10.1093/nar/gks042

McKean M, and Amaria R. 2018. Multidisciplinary treatment strategies in high-risk resectable melanoma: Role of adjuvant and neoadjuvant therapy. Cancer treatment reviews 70:144-153. 10.1016/j.ctrv.2018.08.011

Meng Y, Hertel N, Ellis J, Morais E, Johnson H, Philips Z, Roskell N, Walker A, and Lee D. 2018. The costeffectiveness of nivolumab monotherapy for the treatment of advanced melanoma patients in England. The European journal of health economics : HEPAC : health economics in prevention and care 19:1163-1172. 10.1007/s10198-018-0964-4

Prensner J, and Chinnaiyan A. 2011. The emergence of lncRNAs in cancer biology. Cancer discovery 1:391-407. 10.1158/2159-8290.cd-11-0209

Qi X, Zhang D, Wu N, Xiao J, Wang X, and Ma W. 2015. ceRNA in cancer: possible functions and clinical implications. Journal of medical genetics 52:710-718. 10.1136/jmedgenet-2015-103334

Reddy B, Miller D, and Tsao H. 2017. Somatic driver mutations in melanoma. Cancer 123:2104-2117. 10.1002/cncr.30593

Robert C, Long GV, Brady B, Dutriaux C, Maio M, Mortier L, Hassel JC, Rutkowski P, McNeil C, Kalinka-Warzocha E, Savage KJ, Hernberg MM, Lebbe C, Charles J, Mihalcioiu C, Chiarion-Sileni V, Mauch C, Cognetti F, Arance A, Schmidt H, Schadendorf D, Gogas H, Lundgren-Eriksson L, Horak C, Sharkey B, Waxman IM, Atkinson V, and Ascierto PA. 2015. Nivolumab in previously untreated melanoma without BRAF mutation. N Engl J Med 372:320-330. 10.1056/NEJMoa1412082

Robinson M, McCarthy D, and Smyth G. 2010. edgeR: a Bioconductor package for differential expression analysis of digital gene expression data. Bioinformatics (Oxford, England) 26:139-140. 10.1093/bioinformatics/btp616

Shen J, Hong L, Yu D, Cao T, Zhou Z, and He S. 2019. LncRNA XIST promotes pancreatic cancer migration, invasion and EMT by sponging miR-429 to modulate ZEB1 expression. The international journal of biochemistry \& cell biology 113:17-26. 10.1016/j.biocel.2019.05.021

Slattery M, Mullany L, Sakoda L, Wolff R, Samowitz W, and Herrick J. 2018. Dysregulated genes and miRNAs in the apoptosis pathway in colorectal cancer patients. Apoptosis : an international journal on programmed cell death 23:237-250. 10.1007/s10495-018-1451-1

Swick J, and Maize J. 2012. Molecular biology of melanoma. Journal of the American Academy of Dermatology 
56

67:1049-1054. 10.1016/j.jaad.2011.06.047

Topalian SL, Taube JM, Anders RA, and Pardoll DM. 2016. Mechanism-driven biomarkers to guide immune checkpoint blockade in cancer therapy. Nat Rev Cancer 16:275-287. 10.1038/nrc.2016.36

Wahid M, Jawed A, Mandal R, Dar S, Akhter N, Somvanshi P, Khan F, Lohani M, Areeshi M, and Haque S. 2018. Recent developments and obstacles in the treatment of melanoma with BRAF and MEK inhibitors. Critical reviews in oncology/hematology 125:84-88. 10.1016/j.critrevonc.2018.03.005

Wilusz J, Sunwoo H, and Spector D. 2009. Long noncoding RNAs: functional surprises from the RNA world. Genes \& development 23:1494-1504. 10.1101/gad.1800909

Xiao P, Liu W, and Zhou H. 2016. miR-429 promotes the proliferation of non-small cell lung cancer cells via targeting DLC-1. Oncology letters 12:2163-2168. 10.3892/ol.2016.4904

Xu ZY, Zhao M, Chen W, Li K, Qin F, Xiang WW, Sun Y, Wei J, Yuan LQ, Li SK, and Lin SH. 2020. Analysis of prognostic genes in the tumor microenvironment of lung adenocarcinoma. PeerJ 8:e9530. 10.7717/peerj.9530

Yang J, Liu Y, He A, Liu Y, Wu J, Liao X, Lv Z, Wang F, and Mei H. 2017. Hsa-miR-429 promotes bladder cancer cell proliferation via inhibiting CDKN2B. Oncotarget 8:68721-68729. 10.18632/oncotarget.19878

Yasui K, Izumida M, Nakagawa T, Kubo Y, Hayashi H, Ito T, Ikeda H, and Matsuyama T. 2018. MicroRNA-3662 expression correlates with antiviral drug resistance in adult T-cell leukemia/lymphoma cells. Biochemical and biophysical research communications 501:833-837. 10.1016/j.bbrc.2018.04.159

Yu G, Wang L, Han Y, and He Q. 2012. clusterProfiler: an R package for comparing biological themes among gene clusters. Omics : a journal of integrative biology 16:284-287. 10.1089/omi.2011.0118

Zhang L, Wang Y, Li X, Wang Y, Wu K, Wu J, and Liu Y. 2020. Identification of a Recurrence Signature and Validation of Cell Infiltration Level of Thyroid Cancer Microenvironment. Front Endocrinol (Lausanne) 11:467. 10.3389/fendo.2020.00467 


\section{Figure 1}

Differential genes of in situ and metastatic samples from melanoma patients in the TCGA database.

(A) Flow chart of the bioinformatics analysis. (B) Differential mRNAs, (C) differential IncRNAs, and (D) differential miRNAs. Blue represents in situ melanoma sample highly expressed genes, red represents metastatic samples highly expressed genes, screen |log2 fold change| $>1.0$, p-value $<0.05$. (E) Metastatic samples highly expressed DEmRNAs were subjected to $\mathrm{GO}$ analysis. BP, CC, and MF functionally enriched, bubble map bubble size indicates the number of genes corresponding to this entry, bubble color correlates with $P$. adjust, (F) Metastatic samples highly expressed DEmRNAs are enriched by KEGG to tumorassociated pathways and highly correlated genes, pathway point size represents the number of differential genes it contains, and gene point color correlates with differential multiplicity. 
A

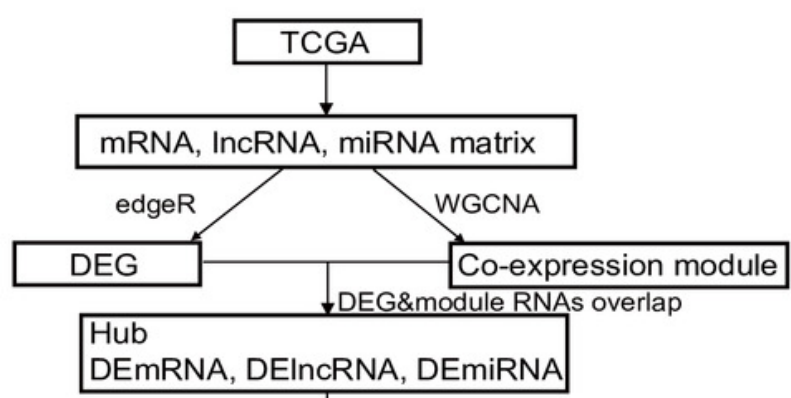

mirDIP, IncBasec database

ceRNA regulation network

Cox, lasso regression, KM survial

Construction of a prognostic model

C

E
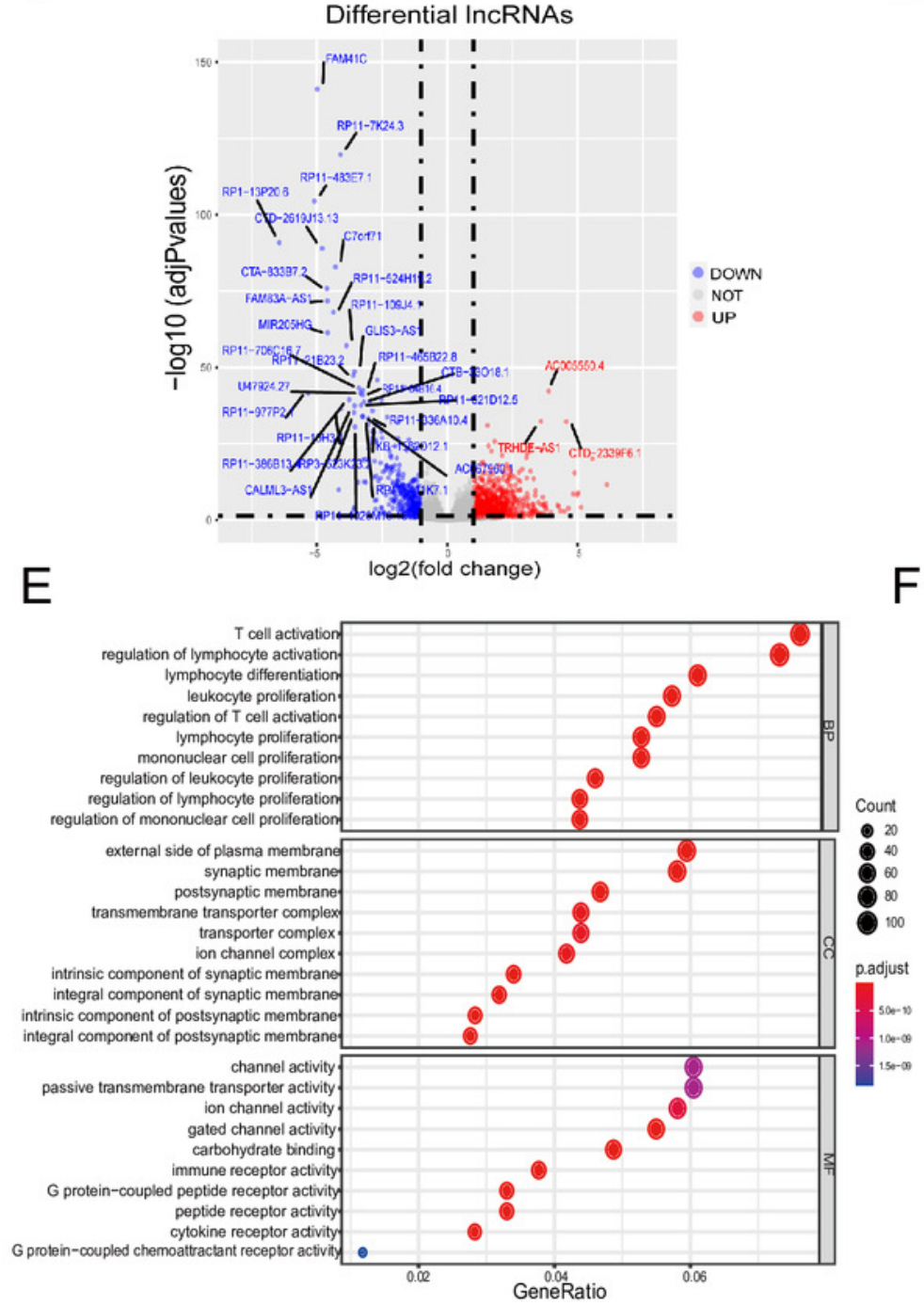

B

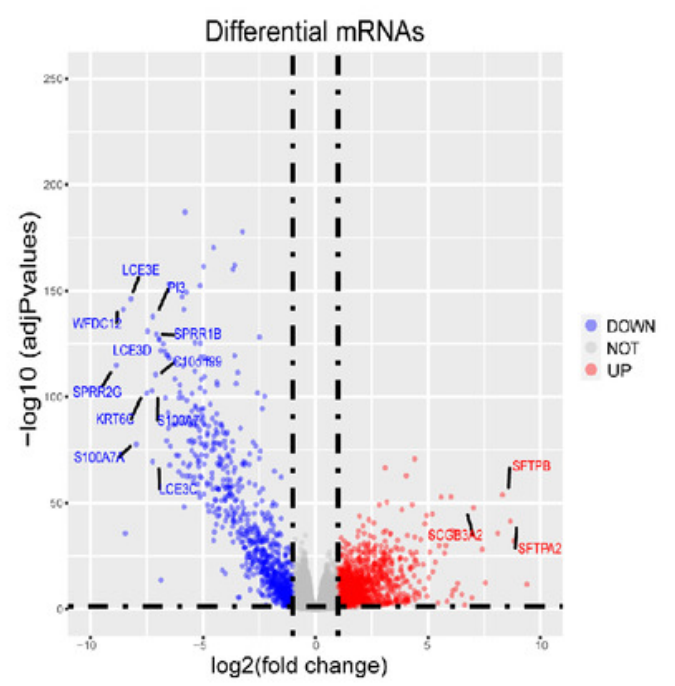

D
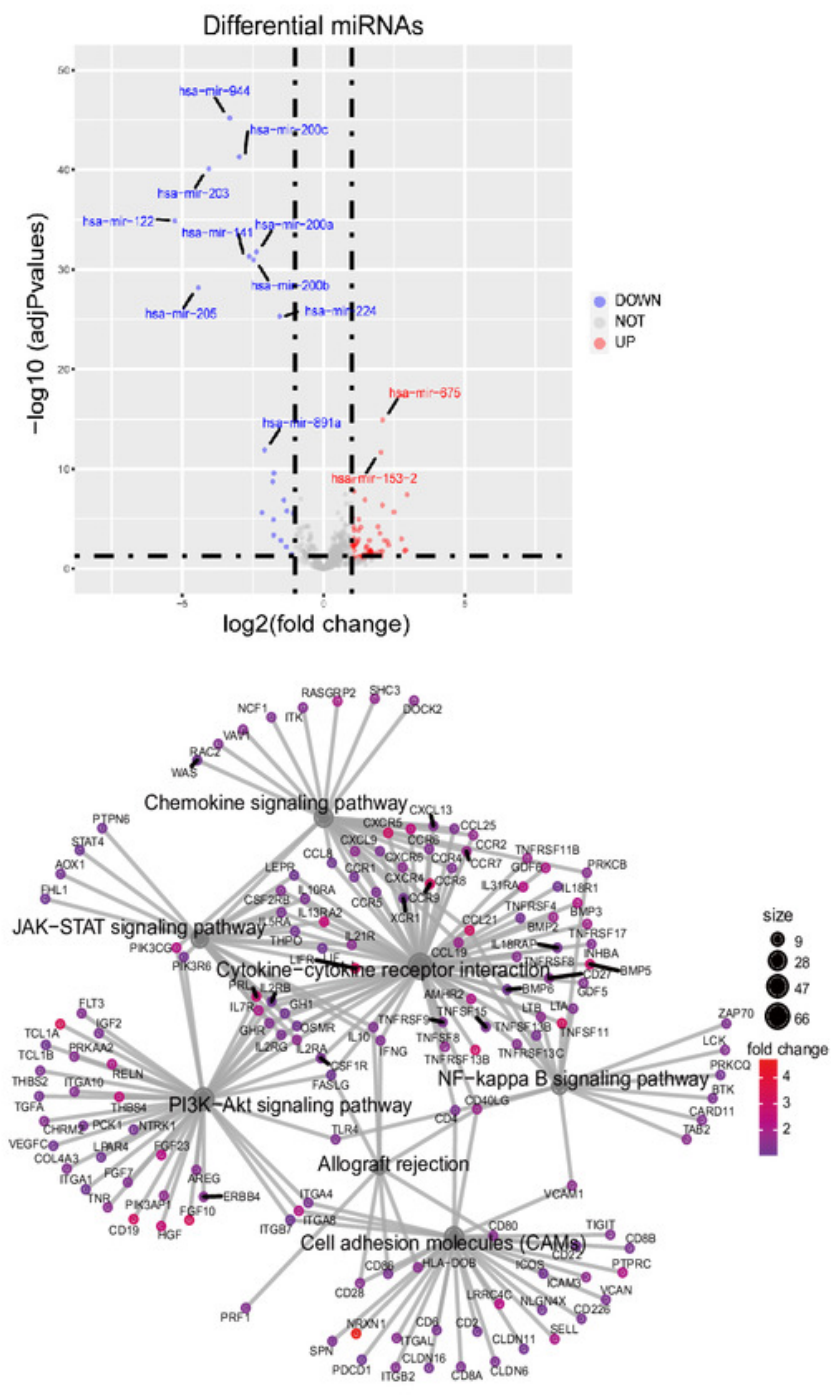


\section{Figure 2}

WGCNA of mRNAs and IncRNAs.

$\square A \square T h e$ scale-free fit index for soft-thresholding powers of mRNA. The soft-thresholding power in the WGCNA was determined based on a scale-free R2 (R2 $=0.95)$. The left panel presents the relationship between the soft-threshold and scale-free R2. The right panel presents the relationship between the soft-threshold and mean connectivity. $\square \mathrm{B} \square \mathrm{A}$ dendrogram of the mRNA clustered based on different metrics. Each branch in the Fig. represents one gene, and every color below represents one co-expression module. $\square С \square A$ heatmap showing the correlation between the mRNA module and clinical traits. The correlation coefficient in each cell represented the correlation between gene module and the clinical traits, which decreased in size from red to blue. $\mathrm{D} \square$ The scale-free fit index for softthresholding powers of IncRNA. $\square \mathrm{E} \square$ A dendrogram of the IncRNA clustered based on different metrics. $\square \mathrm{F} \square \mathrm{A}$ heatmap showing the correlation between the IncRNA module and clinical traits. $\square \mathrm{G} \square$ The 1255 mRNAs in turquoise module were annotated with GO function and important items were selected for drawing DAG $\square$ directed acyclic graph $\square$ graph $\square$ which shows subgraph induced by most significant GO terms. 
A
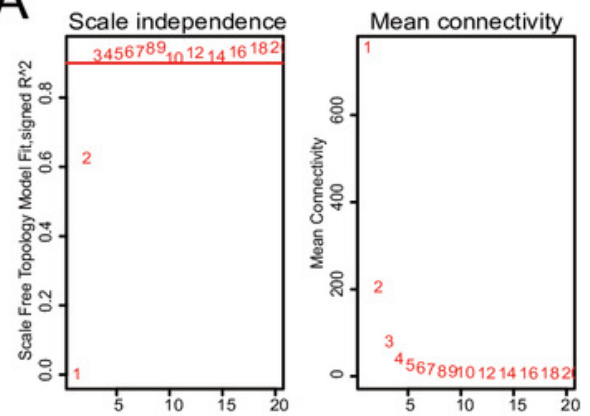

D scale independence
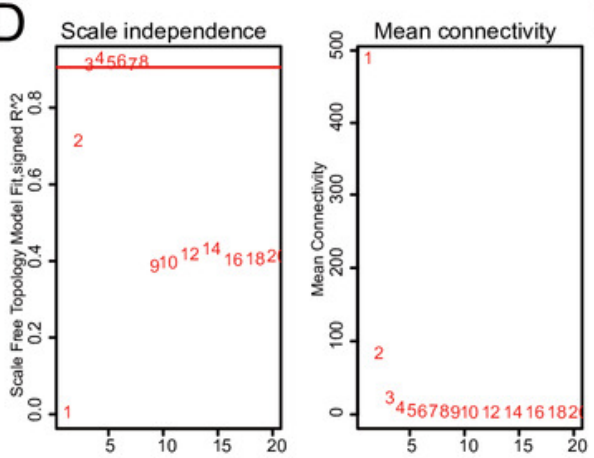

B

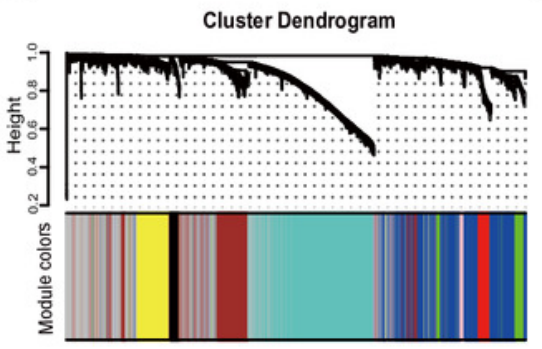

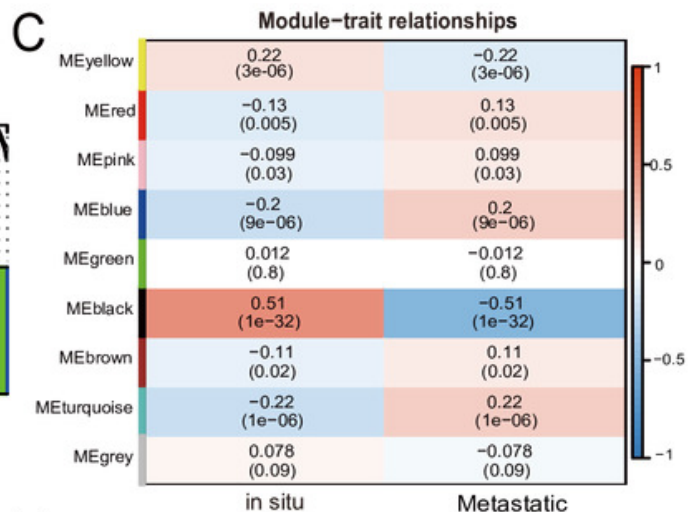

E
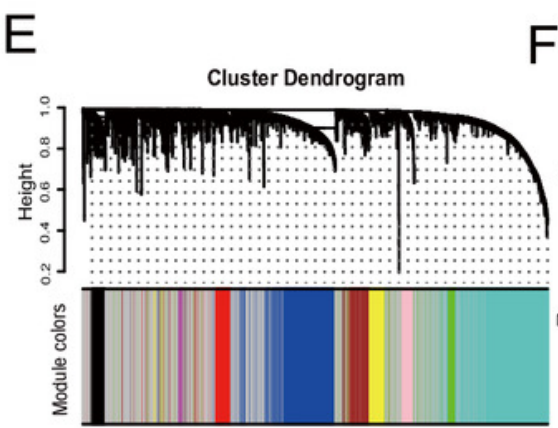

\begin{tabular}{|c|c|c|}
\hline & \multicolumn{2}{|c|}{ Module-trait relationships } \\
\hline MEred & $\begin{array}{c}0.17 \\
(2 \mathrm{e}-04)\end{array}$ & $\begin{array}{l}-0.17 \\
(2 \mathrm{e}-04)\end{array}$ \\
\hline MEblue & $\begin{array}{l}-0.04 \\
(0.4)\end{array}$ & $\begin{array}{l}0.04 \\
(0.4)\end{array}$ \\
\hline MEmagenta & $\begin{array}{c}-0.044 \\
(0.3)\end{array}$ & $\begin{array}{l}0.044 \\
(0.3)\end{array}$ \\
\hline MEblack & $\begin{array}{l}-0.085 \\
(0.07)\end{array}$ & $\begin{array}{l}0.085 \\
(0.07)\end{array}$ \\
\hline MEpink & $\begin{array}{c}0.4 \\
(1 \mathrm{e}-19)\end{array}$ & $\begin{array}{l}-0.4 \\
(1 \mathrm{e}-19)\end{array}$ \\
\hline MEturquoise & $\begin{array}{l}-0.18 \\
(1 \mathrm{e}-04)\end{array}$ & $\begin{array}{l}0.18 \\
(1 \mathrm{e}-04)\end{array}$ \\
\hline MEbrown & $\begin{array}{l}-0.18 \\
(1 \mathrm{e}-04)\end{array}$ & $\begin{array}{l}0.18 \\
(1 \mathrm{e}-04)\end{array}$ \\
\hline MEgreen & $\begin{array}{c}-0.047 \\
(0.3)\end{array}$ & $\begin{array}{l}0.047 \\
(0.3)\end{array}$ \\
\hline MEyellow & $\begin{array}{c}-0.063 \\
(0.2)\end{array}$ & $\begin{array}{l}0.063 \\
(0.2)\end{array}$ \\
\hline MEgrey & $\begin{array}{l}0.11 \\
(0.02) \\
\end{array}$ & $\begin{array}{l}-0.11 \\
(0.02) \\
\end{array}$ \\
\hline & in situ & Metasta \\
\hline
\end{tabular}

\section{G}

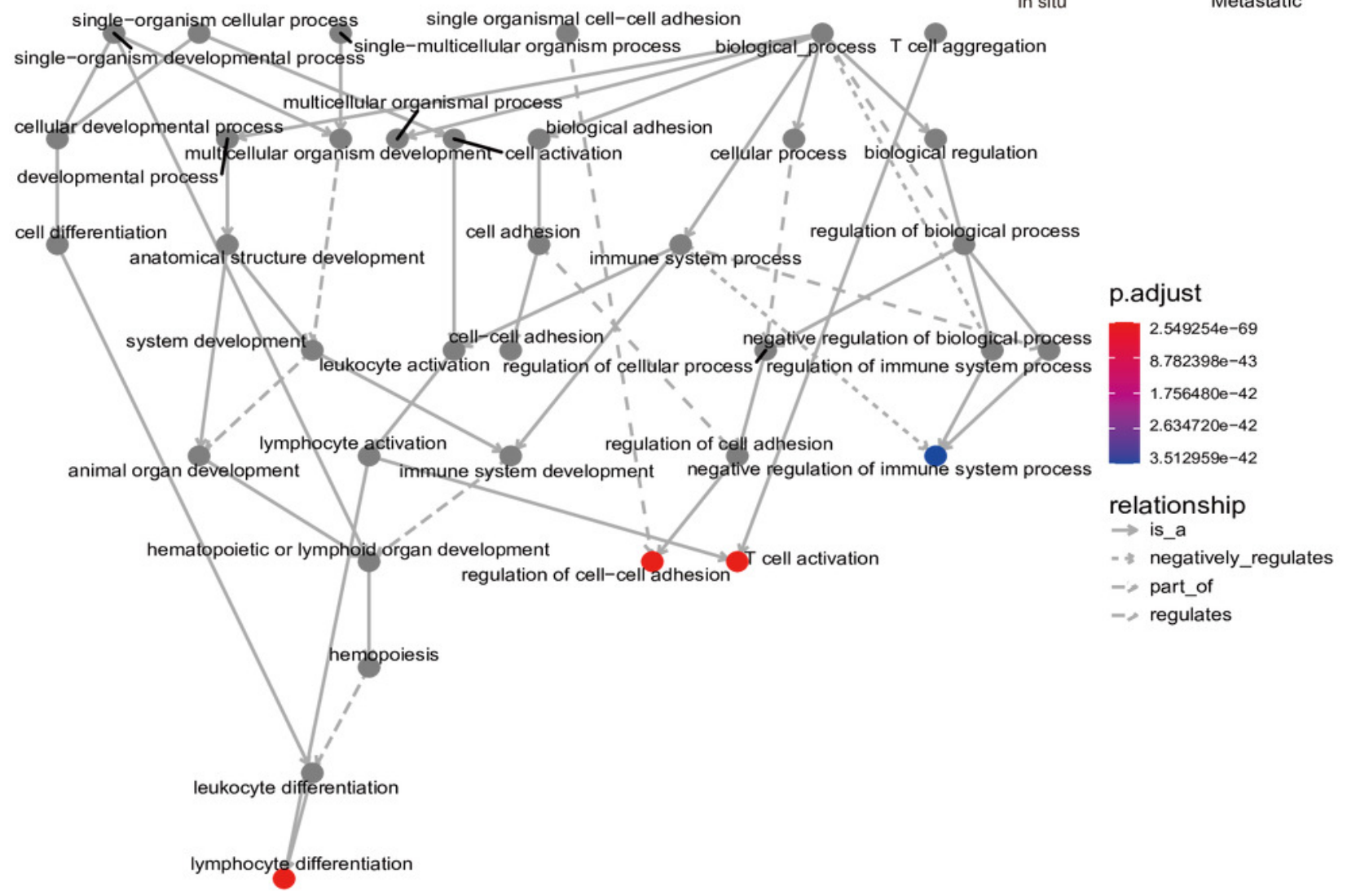




\section{Figure 3}

Selection of hub genes and construction of the ceRNA network.

(A) The mRNAs of turquoise module in WGCNA was intersected with highly expressed mRNA from metastatic samples in the TCGA database to obtain 319 DEmRNAs, (B) the IncRNAs of turquoise module in WGCNA and brown module were intersected with highly expressed IncRNAs from metastatic samples in the TCGA database to obtain 287 DEIncRNAs.(C) The mirDIP database predicted 3771 target genes intersected with 138 highly correlated DEmRNAs, yielding 11 hub mRNAs, (D) 11 hub mRNAs interacting with their corresponding 6 miRNAs in the correlation network. (E) The IncBase database predicts 3760 IncRNAs corresponding to 6 hub miRNAs, and 17 hub IncRNAs were obtained by intersecting with 89 DEInRNAs selected in correlation analysis, (F) 17 hub IncRNAs interacted with 6 hub miRNAs in a network of relationships. (G) T-SNE downscaling of TCGA data with core IncRNAs, and differences in the distribution of transfer samples and in situ samples. (H) Hub ceRNAs interactions network. (I) Differential expression of genes in cohort GSE65904. 


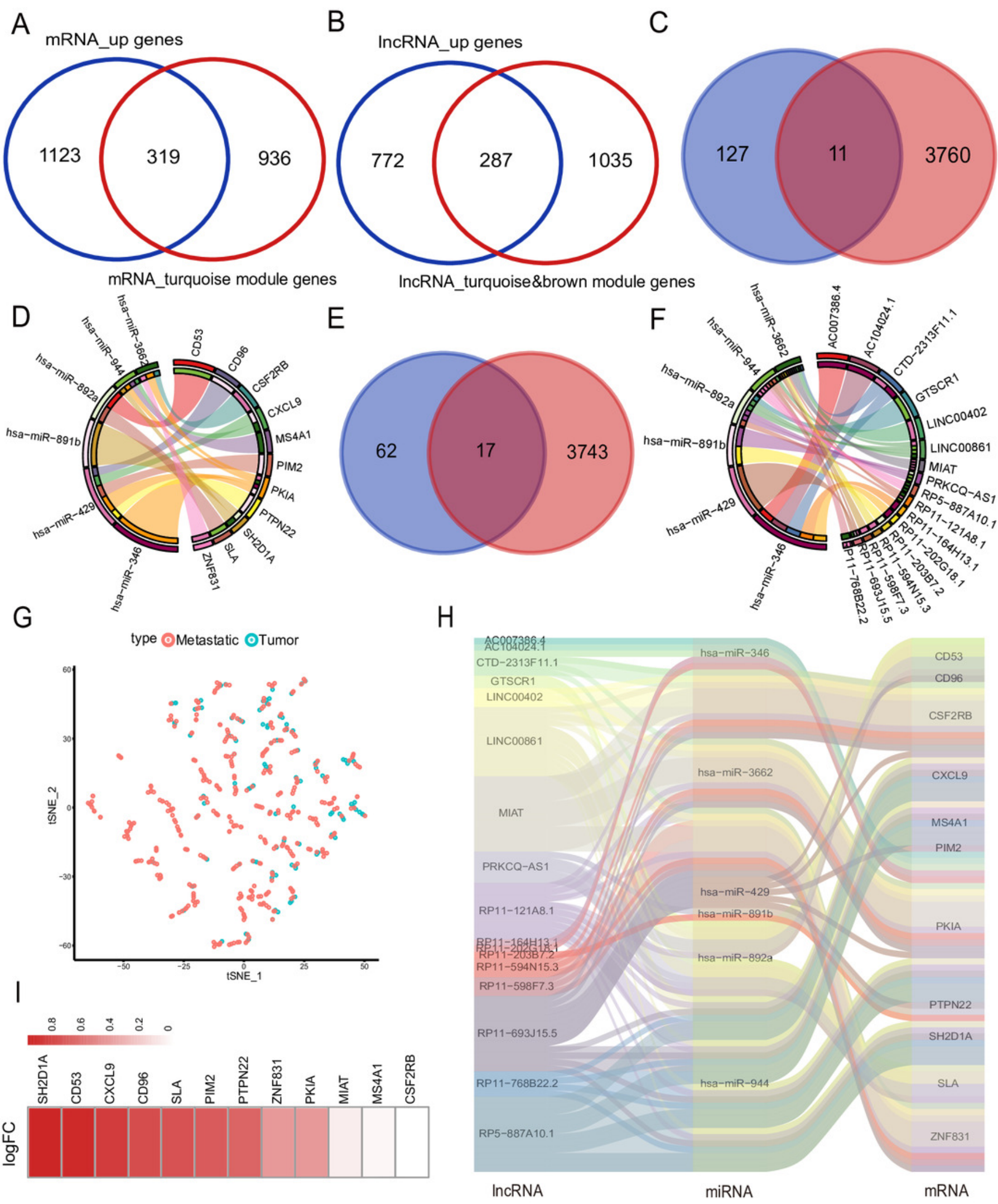




\section{Figure 4}

Construction of metastatic melanoma survival risk prognosis model.

(A, B)Lasso regression of 17 -IncRNAs, when $\lambda=7$, model has the best degree of fitting; $(C$,

D)lasso regression of 6 -miRNAs, when $\lambda=1$, model has the best degree of fitting; $(E, F)$ lasso regression of 11-mRNAs, when $\lambda=2$, model has the best degree of fitting; (G) 10-factors were analyzed using multiple COX regression to construct risk models. 5 of the factors were considered to be risk factors for prognosis and the other five factors were protective factors for prognosis. $(\mathrm{H})$ The distribution of RS; the survival duration and status of patients, and a heatmap of IRGs in the classifier. (I) KM curve of the TCGA cohort according to the expression profiles of risk factors and protective factors in multivariate COX regression models. (J) The 4 groups of ceRNA triplets corresponding to high risk factors. 
A

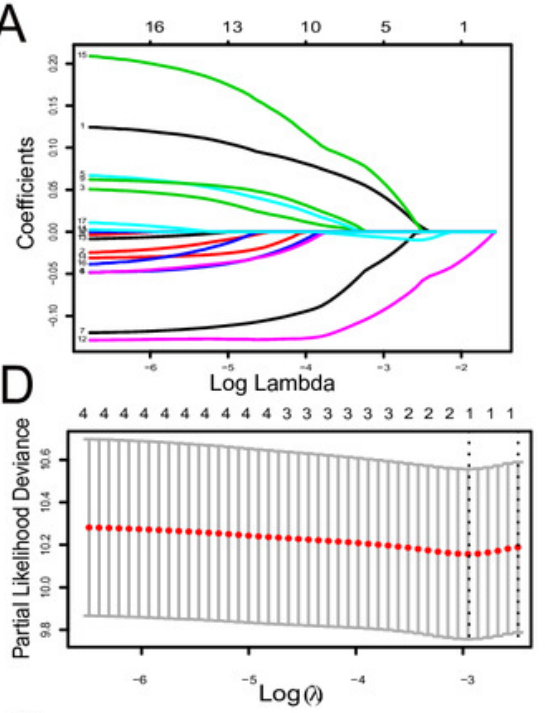

B

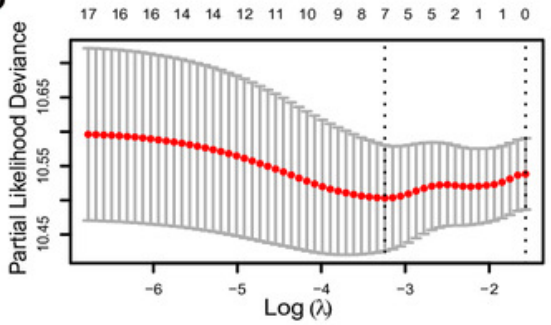

E

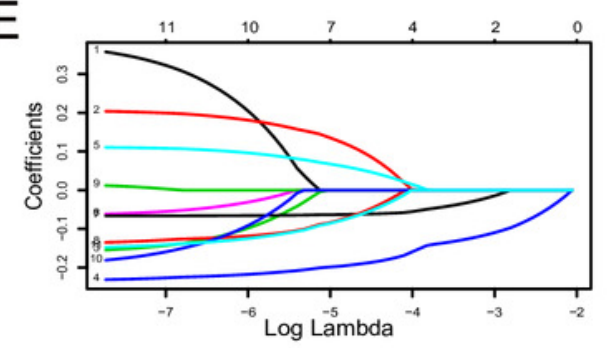

C

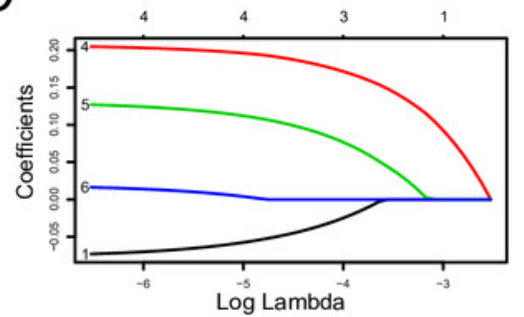

$\mathrm{F}$

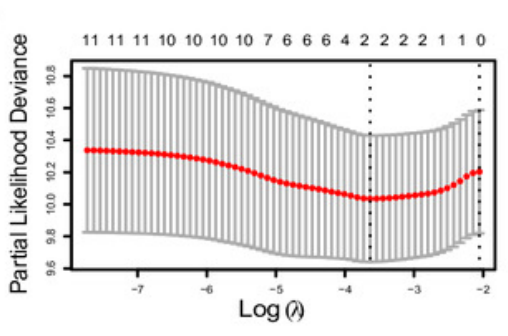

G

\begin{tabular}{|c|c|c|c|c|c|}
\hline & & & & \multicolumn{2}{|c|}{ Concordance Index: 0.7} \\
\hline Variable & & $\mathrm{N}$ & Hazard ratio & & $p$ \\
\hline \multirow[t]{2}{*}{ AC007386.4_group } & low 15 & 153 & & Referenœ & \\
\hline & high 16 & 169 & & $0.88(0.46,1.66)$ & 0.687 \\
\hline \multirow[t]{2}{*}{ AC104024.1_group } & low 17 & 174 & & Referenœ & \\
\hline & high 14 & 148 & & $2.28(1.24,4.19)$ & 0.008 \\
\hline \multirow[t]{2}{*}{ MIAT_group } & low 15 & 156 & & Referenœ & \\
\hline & high 16 & 166 & & $0.38(0.21,0.68)$ & 0.001 \\
\hline \multirow[t]{2}{*}{ RP11.598F7.3_group } & low 16 & 163 & & Referenœ & \\
\hline & high 15 & 159 & & $0.68(0.37,1.26)$ & 0.220 \\
\hline \multirow[t]{2}{*}{ RP11.594N15.3_group } & low 17 & 177 & & Reference & \\
\hline & high 14 & 145 & & $2.78(1.37,5.65)$ & 0.005 \\
\hline \multirow[t]{2}{*}{ RP11.693J15.5_group } & low 15 & 158 & & Referenœ & \\
\hline & high 16 & 164 & & $0.67(0.40,1.14)$ & 0.140 \\
\hline \multirow[t]{2}{*}{ GTSCR1_group } & low 20 & 204 & & Reference & \\
\hline & high 11 & 118 & & $0.85(0.48,1.49)$ & 0.563 \\
\hline \multirow[t]{2}{*}{ CXCL9_group } & low 15 & 150 & & Referenœ & \\
\hline & high 17 & 172 & & $0.99(0.58,1.69)$ & 0.965 \\
\hline \multirow[t]{2}{*}{ hsa.mir.429_group } & low 15 & 151 & & Reference & \\
\hline & high 17 & 171 & $\mathbf{I}$ & $2.26(1.44,3.54)$ & $<0.001$ \\
\hline \multirow[t]{2}{*}{ PKIA_group } & low 17 & 171 & & Referenœe & \\
\hline & high 15 & 151 & & $0.24(0.11,0.52)$ & $<0.001$ \\
\hline
\end{tabular}

।

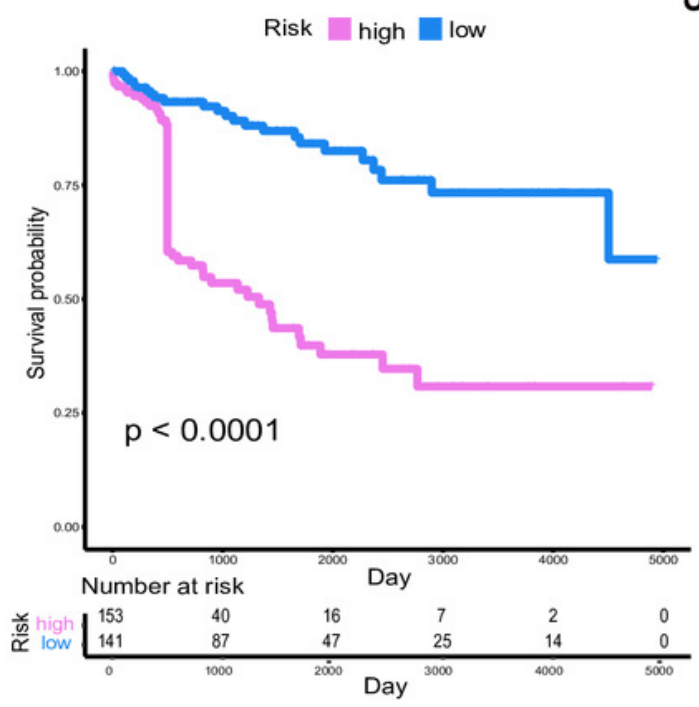

$\mathrm{J}$

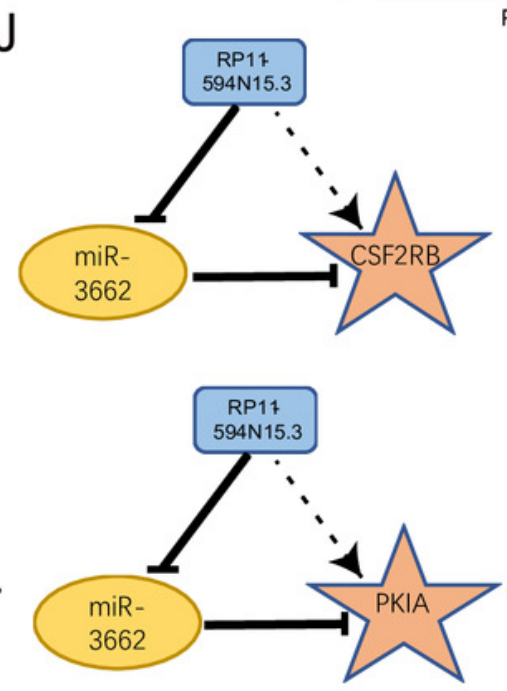

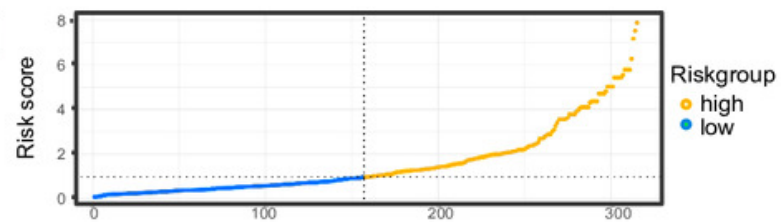
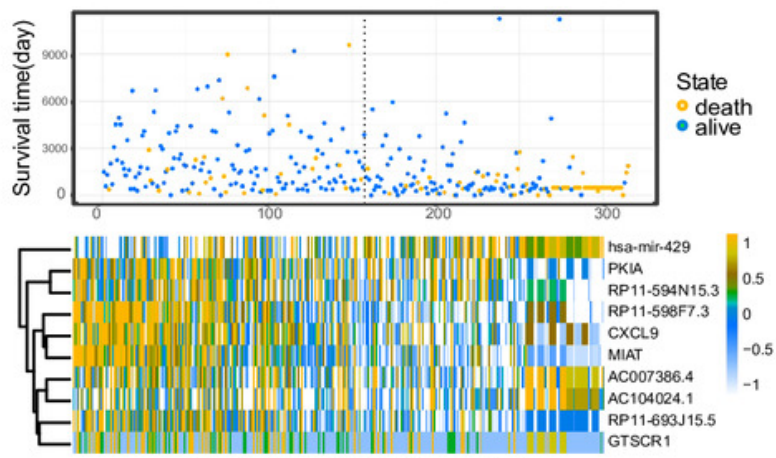

Patient ID(increasing risk score)
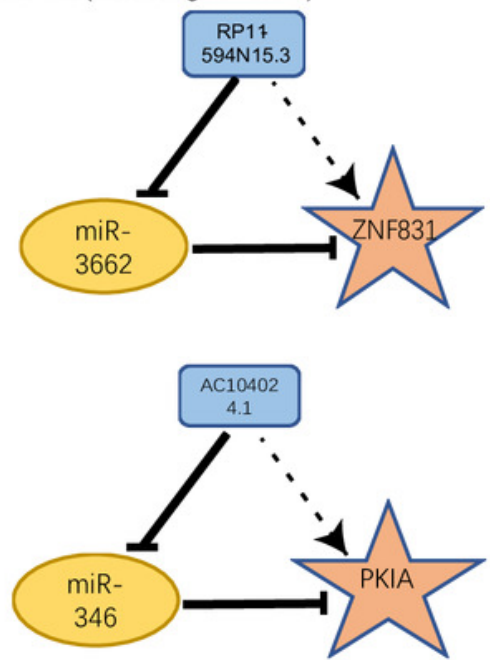


\section{Figure 5}

miR-3662 upregulation suppresses melanoma cell migration and invasion.

(A)Localization of IncRNA RP11-594N15.3 in A375 cells by FISH assay. (B) LncRNA

RP11-594N15.3 was knocked down in A375 cells, qRT-PCR analyses were used to detect its expression level. (C) QRT-PCR analyses were used to detect miR-3662 expression when RP11-594N15.3 was knocked down. (D) QRT-PCR analyses were used to detect the target mRNAs expression when RP11-594N15.3 was knocked down. (E)A375 cells were transfected with plasmids has-mir-3662 and ShNC, qRT-PCR analyses were used to detect miR-3662 expression in transfected cells. (F) QRT-PCR analyses were used to detect the target mRNAs expression in transfected cells. (G) CCK-8 proliferation assay was performed on A375 miR-3662 overexpress and control cells. (H) Transwell cell migration and invasion assays were performed on A375 miR-3662 overexpress and control cells. $* P<0.05, * * P<0.01$, $* * * P$ $<0.001$, $* * * * P<0.001$. 

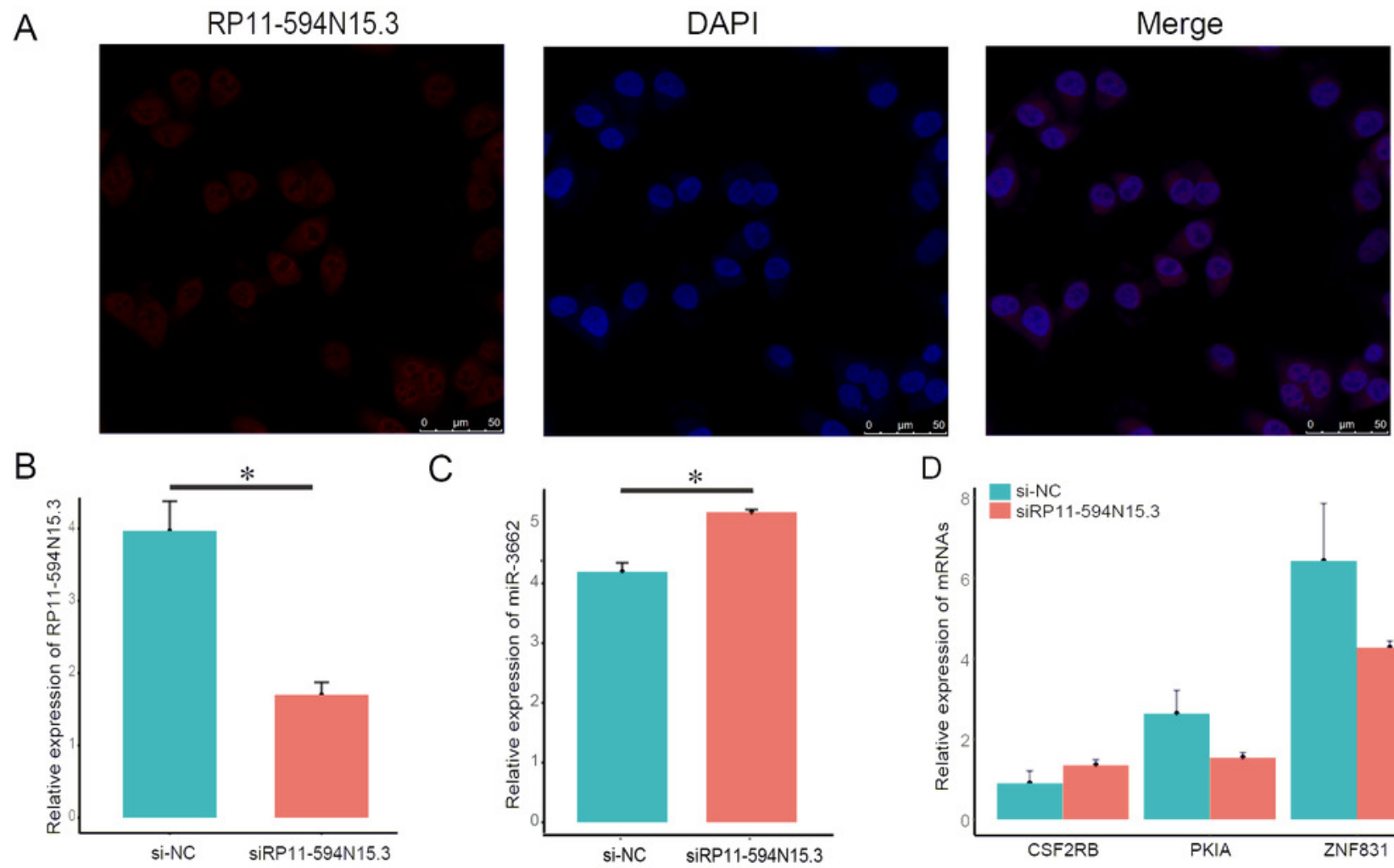

C

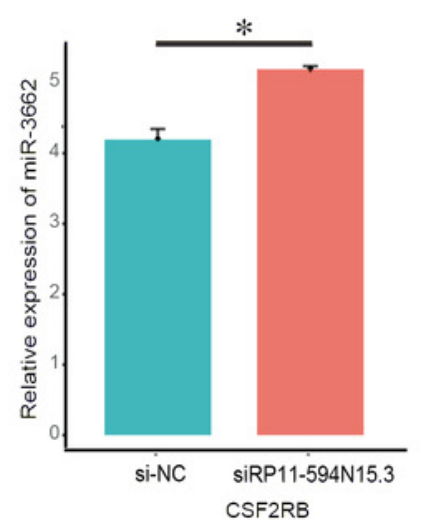

D
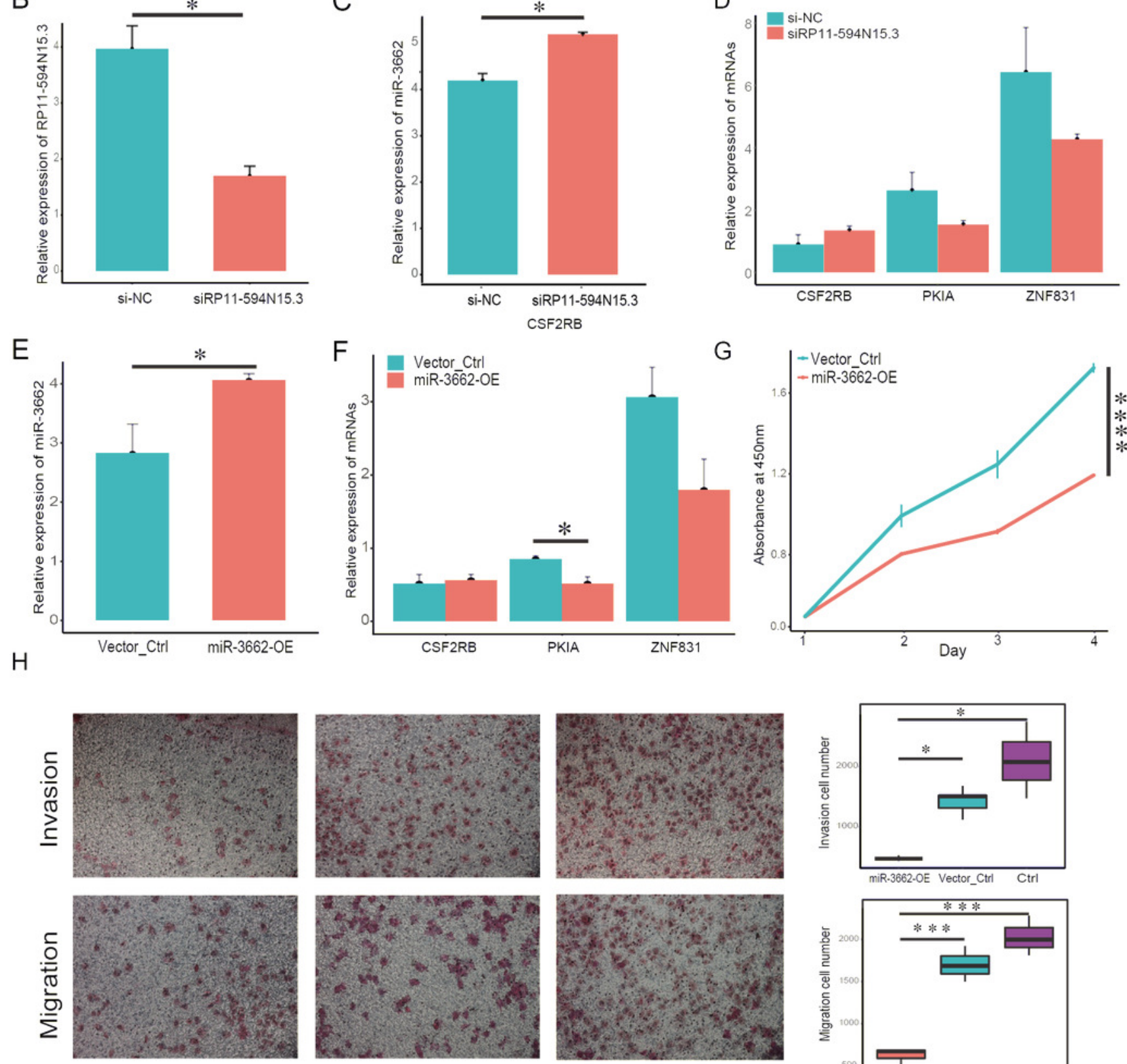

miR-3662-OE

Vector_Ctrl

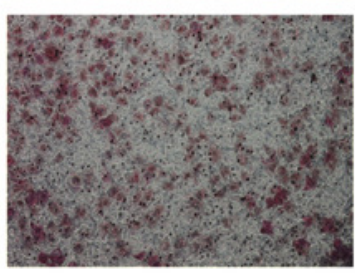

Ctrl
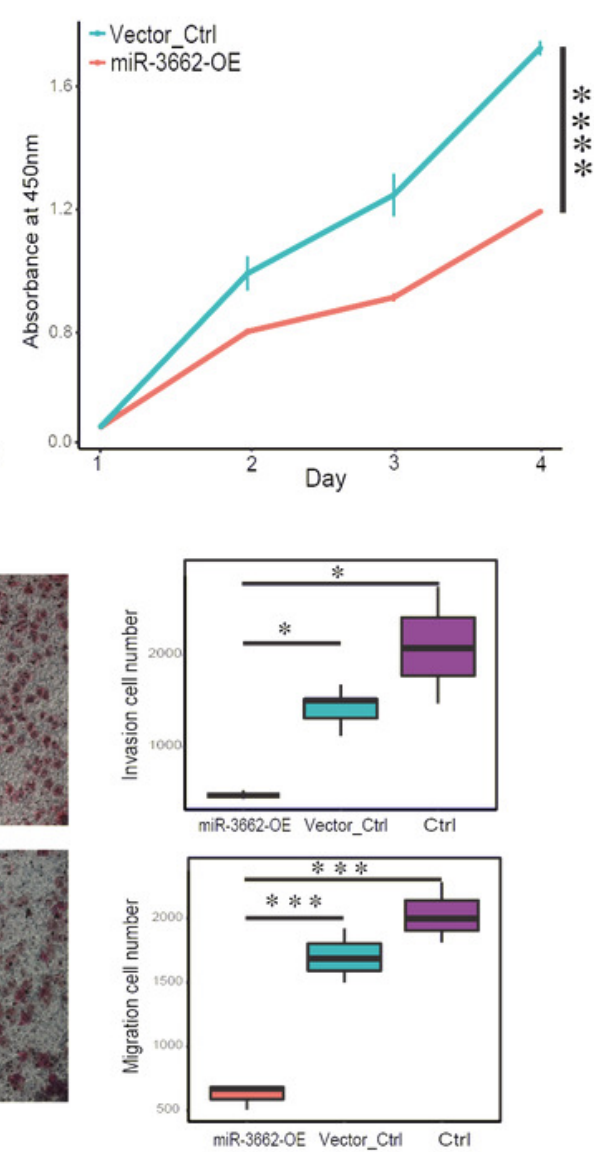